\title{
Nonlinear Analysis Chaotic Characteristics of Leak Signal of Pipeline based on Reconstructed Phase Space
}

\author{
${ }^{1}$ Lei Shi, ${ }^{2, *}$ Jianjun $\mathrm{Xu},{ }^{3}$ Guohui Qu and ${ }^{2}$ Limei Yan \\ ${ }^{1}$ School of Automation, Baotou Light Industry Vocational Technical College, \\ Baotou, 014035, China \\ ${ }^{2}$ College of Electrical Information Engineering, Northeast Petroleum University, \\ Daqing, 163318, China \\ ${ }^{3}$ School of Petroleum Engineering, Northeast Petroleum University, Daqing,163318,P.R. \\ China \\ * Corresponding author: Jianjun Xu, E-mail: 123939274@qq.com
}

\begin{abstract}
The In this paper, nonlinear analysis is used to study the possibility of chaotic behavior of pipeline pressure signal. Based on real-time data of pipeline leak monitoring system, oil pipeline pressure signals have been verified to be chao system by analyzing the chaotic characteristic of pressure time series. Six typical measured data of pipeline pressure are selected and reconstructed to the higher phase space. And then the largest Lyapunov exponent of each data is calculated to test and verify the chaotic characteristics of the pressure signal. For pipeline leakage fault diagnosis, the approximate entropy (ApEn) has been applied to extract the nonlinear and chaotic characteristics. By calculating the ApEn of normal, operation and leakage signals, the results indicate that the value ranges of three kinds of signals are above 0.35, below 0.025, and from 0.025 to 0.35 . And the identification rate of pipeline leakage has been reached $90.0 \%$ only based on ApEn, which provide more effective basis for the classification and identification of chaotic characteristics of the pressure signal.
\end{abstract}

Keywords: Nonlinear chaotic time series, reconstructed phase space, oil pipeline leakage detection

\section{Introduction}

With the rapid development of oil exploration, frequent fluctuations in pipeline pressure are always caused by many complex operating conditions such as multipoint sub-transmission, large drop and sequential transportation[1-2]. Those operating conditions result in more false alarms of pipeline leak detection system occur, and in addition, the leak signal becomes much weaker on account of longer and longer distance between pump stations. In order to quickly identify and accurately locate the leakage, and reduce the false alarm rate further, how to extract the weak leakage signal under the complex working conditions has been a difficult and urgent problem for pipeline leakage detection technology[3-4].

Several decades ago, the method for pipeline leak detection was proposed by the German scholar R.Isermann and H. Siebert with the flow and pressure of inlet and outlet signals processed by cross-correlation analysis. In order to maintain the leakage information detectable and durable, L.Billman and R. Isermann utilized nonlinear state observer to detect the pipeline leakage under different working conditions. Fiber optic interferometer based on Sagnac, wavelet analysis, fuzzy logic, and nondestructive testing had been put forward to apply in pipeline leakage detecting technology. Since the eighties, some of our research institutes and 
universities have obtained fruitful achievements in the stress wave method, negative pressure wave and pipeline real-time model el, al. Pattern recognition and wavelet analysis technique had been used by Jin S. J. and Wang L.N. for improving negative pressure wave largely. Then, according to pressure, flow and differential flow, fuzzy algorithm and logical judgment method were adopted based on negative pressure wave and mass balance principle to detect leak. Sequential probability ratio test was applied to judge and locate the small leakage[5-6]. GPS timing system was combined with negative pressure wave for pipeline leakage monitoring system, and an advanced technology based on the instantaneous pipeline model was proposed by Liu E.B. The model was solved by improved differential scheme of character lines method. In practical engineering applications, pipeline leak detection system based on negative pressure wave method can accurately identify and locate the leak which is greater than 1 percent of the instantaneous flow, and the small leak is still not easy to recognize.

Chaotic time series theory can indicate the nonlinear characteristics of complex system by a single variable time series data, and also has the ability to detect weak signals[7-8]. Therefore, 6 typical pressure signals in the same pipe section are selected as the analysis objects in this paper. The existence of chaotic characteristics of pipeline pressure time series has been researched through the intrinsic properties of the pressure time series.

\section{Analysis of Chaotic Time Series}

\subsection{Reconstruction of Phase Space}

According to the Takens theory [9], the delay-coordinate embedding technique is adopted to reconstruct phase space. A proper embedding dimension $m$ needs to be found to guarantee that a topological equivalence of the phase space of the intrinsic unknown dynamical system can be reconstructed from time series, based on which characteristics of the dynamical invariant set can be estimated. From each measured time series, the following vector quantity of $m$ components is constructed,

$$
X_{i}=\left(x_{t}, x_{t+\tau}, x_{t+2 \tau}, \ldots, x_{t+(m-1) \tau}\right), t=1,2, \ldots, N_{d}
$$

Where $N_{d}=N-(m-1) \tau, \tau$ is time delay, $m$ is embedding dimension.

$\mathrm{m}$ and $\tau$ are key parameters of phase space reconstruction. The choice of an appropriate $\tau$ is critical, and the correlation between the coordinates of delay vectors is measured by $I(\tau)$.

Then $I(\tau)$ can be calculated by:

$$
I(\tau)=\sum_{x_{i}, x_{i+\tau}} P\left(x_{i}, x_{i+\tau}\right)
$$

Mutual information method is that when $I(\tau)$ between each state vector of reconstructed phase space has reached its first minimum value, and then it can be considered that the reconstructed phase space is topological equal to the original one.

An optimal embedding dimension can well show the internal structure of attractors. If $\mathrm{m}$ is too small, the attractors will fold. Conversely, an excessive $\mathrm{m}$ not only exponentially increases the calculation, but also causes a lot of errors in the system with noise. Based on the improved false nearest algorithm, only one parameter $\tau$ can be used to solve the optimal $\mathrm{m}$ by Cao method with small data. Essentially, when the phase space dimension increases from $m$ to $m+1$, it needs to find the false nearest points which is adjacent in the low dimension space reflected from nonadjacent in the high dimension space. When the ratio of false nearest points 
no longer decreases with increasing $\mathrm{m}$, it can be considered as attractors have been fully expanded. The false nearest point is selected by:

$$
b(i, m)=\frac{\left\|x_{m+1}(i)-x_{m+1}^{N N}(i)\right\|}{\left\|x_{m}(i)-x_{m}^{N N}(i)\right\|}
$$

Where $x_{m}(i)$ and $x_{m}{ }^{N N}(i)$ are the $\mathrm{i}$-th phasor and its nearest point in the $m$ dimension space.

The optimal embedding dimension $m_{0}$ is:

$$
E(m)=\frac{1}{N-m \tau} \sum_{i=1}^{N-m \tau} b(i, m) \quad E^{\prime}(m)=\frac{E(m+1)}{E(m)}
$$

If time series is determined, when $\mathrm{m}$ has been larger than $\mathrm{m} 0$ and the differences of $E^{\prime}(m)$ convergent to the mean of differences under $0.1, \mathrm{~m} 0$ is the optimal embedding dimension.

\subsection{Correlation Dimension D}

GP algorithm can be used to obtain the correlation dimension D and it's first proposed by Grassberger and Procacciay[10-11].

$$
\begin{gathered}
C_{n}(r)=\frac{1}{M^{2}} \sum_{i, j=1}^{M} \theta[r-\|X(i)-X(j)\|] \\
D=\lim _{r \rightarrow 0} \frac{\ln C_{n}(r)}{\ln r}
\end{gathered}
$$

Where $\|X(i)-X(j)\|$ is distance between two points of phase space, correlation integral $C_{n}(r)$ shows the probability of distance between two vectors in the reconstructed phase space less than $\mathrm{r}$; $\mathrm{M}$ is the number of phase points; $\theta(x)$ is Heaviside function.

Based on GP algorithm, the slope of the liner part of double-log curve of $\ln C_{n}(r)-\ln r$ is the correlation dimension of this time series.

\subsection{The Largest Lyapunov Exponent}

The primary characteristic of chaotic systems is extremely sensitive to initial condition. The orbits generated by two neighboring initial value exponentially separate with time. As an important characteristic of chaotic system, the largest Lyapunov exponent (referred as LLE) determines whether the time series has chaotic property. Usually the system is considered chaotic when LLE is greater than zero. Small data set is always used for calculating LLE[12-13].

Step1: On the basis of reconstruction of phase space, the mean period $\mathrm{P}$ of time series is calculated by FFT. On a given orbit the nearest neighboring point of each point needs to be found and restricted on temporary separation. That is:

$$
d_{j}(0)=\min _{X_{i}}\left\|X_{j}-X_{\hat{j}}\right\|,|j-\hat{j}|>P
$$

Step 2: The distance of the $j$-th nearest neighbors after i discrete time steps on the basic tracks can be calculated by

$$
d_{j}(i)=\left\|X_{j+i}-X_{\hat{j}+i}\right\|
$$

Where $\mathrm{i}=1,2, \ldots, \min (M-j, M-\hat{j})$.

Step 3: The average of all the $j^{`} \ln d j(i)$ for each $i$ is given by 


$$
p(i)=\frac{1}{l \Delta t} \sum_{j=1}^{l} \ln d_{j}(i)
$$

Where $l$ is the number of $d_{j}(i)$ without zero.

Step 4: The least squares method is applied to solving the slope of regression line, which is LLE.

\subsection{Approximate Entropy}

The chaotic characteristics of signals can be displayed by nonlinear chaotic indicators greatly. Except for the correlation dimension and LLE, nonlinear characteristic is a notable character of approximate entropy (referred as ApEn) .

One-dimension vector has been reconstructed as definition (1). $d[X(i), X(j)]$ is thought as the largest one of the difference between $X(i)$ and $X(j) . C_{i}^{m}(r)$ is defined as the proportion of the number of $d[X(i), X(j)]$ that is smaller than the given threshold $r$ for each $i$ in the amount of distance N-m. We can get:

$$
\Phi^{m}(r)=(N-m+1)^{-1} \sum_{i=1}^{N-m+1} \ln \left[C_{i}^{m}(r)\right]
$$

Then the ApEn is:

$$
\operatorname{ApEn}(m, r, N)=\Phi^{m}(r)-\Phi^{m+1}(r)
$$

Essentially, when the dimension increases from $m$ to $m+1$, the probability of new model and the ratio of new information of the time series is reflected by ApEn. The value of ApEn increased with the rising of signal complexity. Therefore, ApEn is used to represent the overall characteristics of the signal from the view of measuring the complexity of the time series. Compared with the D and LLE, ApEn algorithm does not attempt to completely reconstructed attractor, but calculate each segment from a statistical point. As a characteristic indicator of chaotic time series it has no demand of the length of the data volume and also has some anti-noise ability ${ }^{[14-16]}$.

\section{Case Study}

The experimental data in this paper is collected from the pressure signal of QingTie old pipeline between Lishu and Changtu station. And the length of the pipeline is $68.7 \mathrm{~km}$, the diameter is $\Phi 720 \mathrm{~mm}$, and the pressure is about $1.0 \mathrm{MPa}$. The simulation leak was carried out in the pipe section, and the pipeline pressure signals of each sub-station are sent to center station of leak detection system. The sample frequency of the data is $20 \mathrm{~Hz}$ and 2400 points (data set in 2 minutes) are regarded as a time series[17].

Three kinds of signal including normal operation, processing operation and pipeline leakage are showed respectively in the figure 1. Two time series of each kind of signals have been selected for analysis and comparison of chaotic characteristics as A to F. 


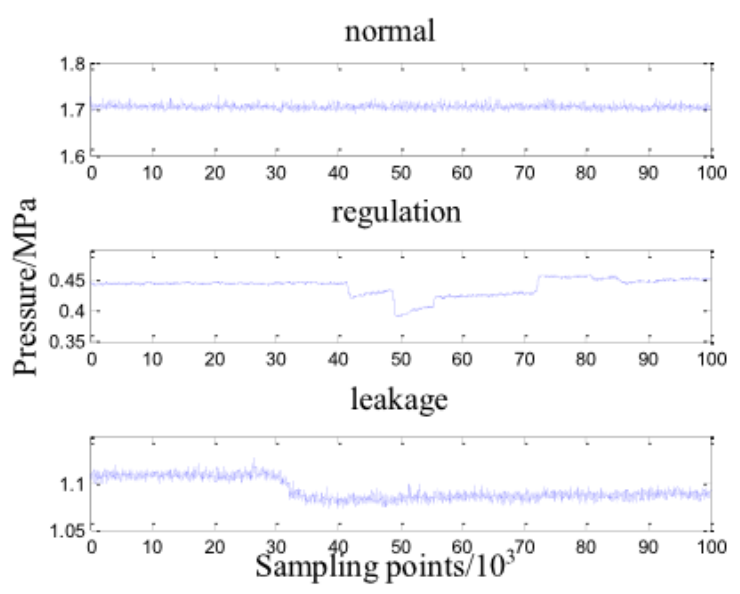

Figure 1. Pipeline Pressure Signals

According to the theory above, the chaotic characteristics of six typical pressure time series are calculated. Mutual information is used for time delay as shown in figure 2 , in which all the smooth curves convergence to the minimum immediately.

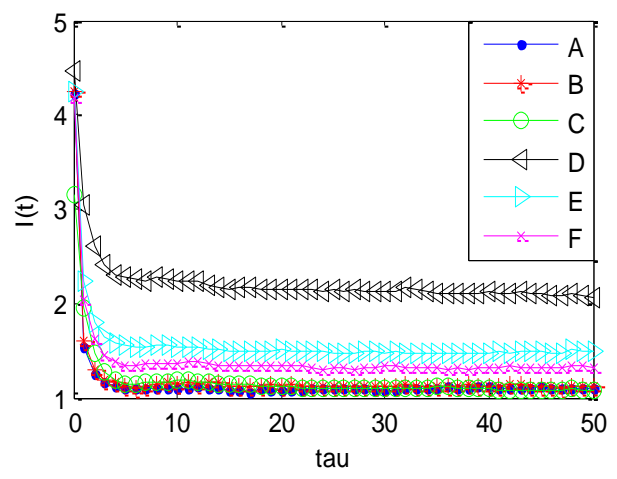

Figure 2. Mutual Information for Delay

The optimal embedding dimension of a time series is given by Cao method. As shown in figure $3, E 1(m)$ tends to a fixed value while $m$ is larger than 8 .

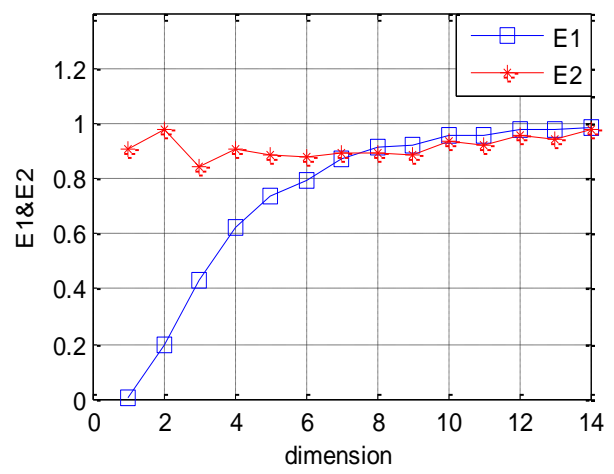

Figure 3. Cao for Embedding Dimension 

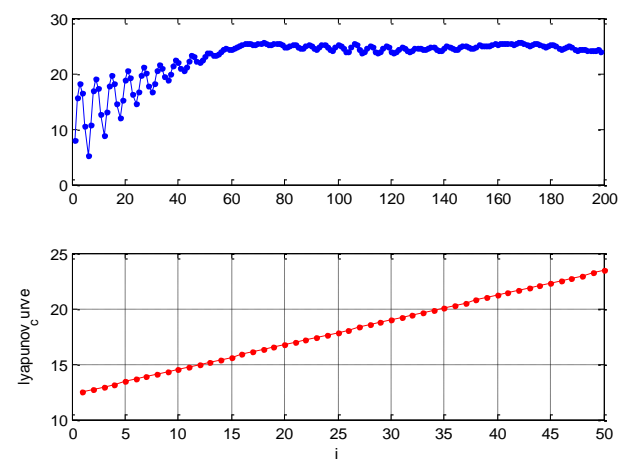

\section{Figure 4. The ApEn of Pipeline Pressure Signals}

LLE is calculated by Kant's small data method, and its curve and slope lines are shown in the figure 4. It can be seen from the lower part of figure 4, LLE of the time series is 0.2223 . It represents that pipeline pressure time series has chaotic characteristics because LLE is larger than zero. The correlation dimension was calculated based on GP algorithm and the results are shown in Table 1. All the correlation dimensions are fractional and also theoretical satisfy $m \geq 2 D+1$. The correlation dimensions of three signals are stable in their appropriate ranges, and the internal complexity of pipeline pressure signals are indicated clearly by D. Among all correlation dimensions, the one of normal operation signal is the largest one and it has strong chaotic performance. In contrast, the regularities of operation and leakage signals are more obvious[18-20].

\section{Table 1. Chaotic Characteristics of Pressure Signals}

\begin{tabular}{|c|c|c|c|c|c|}
\hline \multicolumn{2}{|c|}{ Type of signal } & tau & $\mathrm{m}$ & $\mathrm{D}$ & LLE \\
\hline \multirow{2}{*}{ normal } & $\mathrm{A}$ & 6 & 11 & 4.5915 & 0.2223 \\
& $\mathrm{~B}$ & 6 & 11 & 4.3120 & 0.2001 \\
\hline \multirow{2}{*}{ regulation } & $\mathrm{C}$ & 6 & 9 & 1.9452 & 0.2160 \\
& $\mathrm{D}$ & 7 & 8 & 2.2400 & 0.1855 \\
\hline \multirow{2}{*}{ leakage } & $\mathrm{E}$ & 6 & 7 & 2.9279 & 0.2682 \\
& $\mathrm{~F}$ & 6 & 7 & 2.9938 & 0.2474 \\
\hline
\end{tabular}

The twenty minutes continuous pressure data between Lishu and Changtu section have been collected. They are in four conditions (normal operation, regulating operation, sudden and slow leakage). The corresponding ApEn has been calculated and shown in the figure 5 , with the threshold setting $0.25^{*}$ standard deviation.
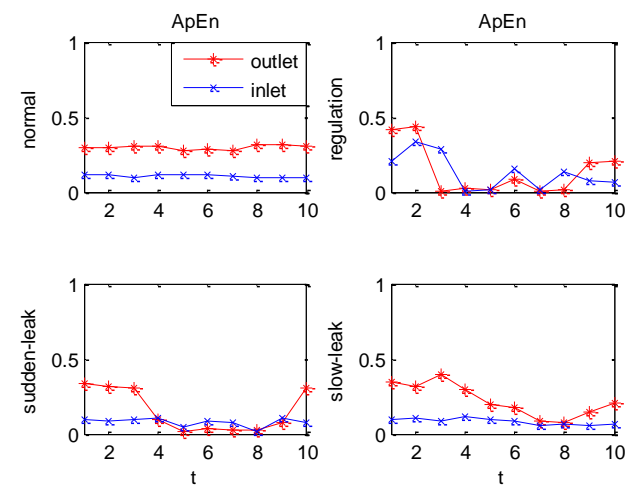

Figure 5. The ApEn of Pipeline Pressure Signals 
As known from figure 5, the inner dynamic chaotic degrees of the fluid decrease differently between pipeline regulation and leakage ${ }^{[21]}$. Firstly, the normal signal ApEn of inlet and outlet are both steady and largest than any other signal, so that the complexity of normal signal is most obvious. Secondly, when the regulation operations happened such as adjusting pump and valve, the inner regularity of oil flow is better than before, thus the outlet ApEn falls sharply. When the leakage fault occurred in the oil pipeline, the chaotic degree of outlet decreases less than regulation operation with the sudden leakage greater than $3 \%$ but the inlet is almost unaffected. In addition, when the slow leak was between $1 \%$ and $3 \%$, the ApEn trends of outlet and inlet are similar to the sudden leakage and more weaken, but the value characteristics are still be able to be observed.

Furthermore, in order to test the performance capability of chaotic characteristics of the pipeline pressure time series by ApEn, 10 more groups of time series which are as the same as above four cases are provided, and No. 1 10 are the normal signal, No. 11 20 are operation signal, No. 21 25 and No. 26 30 are sudden and slow leak signal separately. After calculating and analyzing the ApEn of every condition, the results are shown in Table 2 .

Table 2. The ApEn Value of Three Kinds of Signal

\begin{tabular}{|c|c|c|c|c|c|c|c|c|}
\hline normal & ApEn & result & regulation & ApEn & result & leakage & ApEn & result \\
\hline 1 & 0.2924 & 1 & 11 & 0.0011 & 2 & 21 & 0.0966 & 3 \\
2 & 0.2937 & 1 & 12 & 0.0208 & 2 & 22 & 0.0197 & 2 \\
3 & 0.3080 & 1 & 13 & 0.0155 & 2 & 23 & 0.0365 & 3 \\
4 & 0.3075 & 1 & 14 & 0.0838 & 3 & 24 & 0.0254 & 3 \\
5 & 0.2770 & 1 & 15 & 0.0062 & 2 & 25 & 0.0261 & 3 \\
6 & 0.2896 & 1 & 16 & 0.0143 & 2 & 26 & 0.0833 & 3 \\
7 & 0.2739 & 1 & 17 & 0.0195 & 2 & 27 & 0.0719 & 3 \\
8 & 0.3162 & 1 & 18 & 0.0080 & 2 & 28 & 0.0933 & 3 \\
9 & 0.3162 & 1 & 19 & 0.0698 & 3 & 29 & 0.0901 & 3 \\
10 & 0.3006 & 1 & 20 & 0.0222 & 2 & 30 & 0.1071 & 3 \\
\hline
\end{tabular}

From Table 2 we can draw a conclusion that the ApEn value ranges of the normal signal, the operation signal and the leak signal are above 0.35 , below 0.025 , and 0.025 to 0.35 separately. Recognition rate of pipeline leakage has been reached 90.0\% only based on ApEn. Thus more effective basis for the classification and identification of chaotic characteristics of the pressure signals will be provided. The combination of the ApEn and chaotic characteristics can be used for describing the nonlinearity of pipeline signals more exactly, and the recognition accuracy of the leakage especially slow leak has been improved effectively.

\section{Conclusion}

Based on real-time data of pipeline leak monitoring system, oil pipeline pressure signals have been verified to be chao system by analyzing the chaotic characteristic of pressure time series.

The ApEn has been introduced to the nonlinearity analysis of pressure time series. The inner dynamic evolutions of pipeline flow are described clearly when occurs regulating operation and leakage. The ApEn theory may be used in pipeline leak fault real-time detection system because of its small calculation amount.

(3)Combining the D, LLE and ApEn, the nonlinear characteristics of pressure signal have been expressed more exactly, especially for slow leak signal of pipeline. 
Therefore, an available method for the research on pipeline leak detection has been provided by chaos theory.

\section{Conflict of Interest}

Authors declared that there is no conflict of interest.

\section{Acknowledgement}

This work was supported by Scientific Research Fund of Inner Mongolia Education Department (NO: 12541071).

\section{References}

[1] C. Q.Yuan, X. F. Pang and Y. Liu, "Status and prospect of pipeline leakage detection and location", Journal of Daqing Petroleum Institute, vol. 30, no. 3, (2006), pp. 76-79, 85.

[2] H. Siebert, "A simple method for detecting and locating small leaks in gas pipelines", Process Autom, (1981), pp. 90- 95.

[3] L. M. Yan, Y. S. Zhu and J. J. Xu, "Transmission Lines Modeling Method Based on Fractional Order Calculus Theory", Transactions of China Electrotechnical Society, vol. 29, no. 9, (2014), pp. 260-268

[4] J. P. Kumer, S. A. Kingsley, J. S. Laudo and S. J. Krak, "Applicability of a novel distributed fibre optic acoustic sensor for leak detection”, Proceedings SPIE, vol. 1797, (1993), pp. 63-71.

[5] M. Ferrante and B. Brunone, "Pipe system diagnosis and leak detection by unsteady-state tests", Advances in Water Resources, vol. 26, (2003), pp. 95-105

[6] H. V. da Silva and C. K. Morooka, "Leak detection in petroleum pipelines using a fuzzy system", Journal of Petroleum Science and Engineering, vol. 49, (2005), pp. 223-238.

[7] V. E. Loskutov and A. F. Matvienko, "The magnetic method for in-tube nondestructive testing of gas and oil pipelines: The past and the present", vol. 42, no. 8, (2006), pp. 493-504.

[8] D. L. Yu and S. J. Zhang, "Product oil pipeline leak monitoring technology and project implementation", Computer Measurement \& Control, vol. 19, no. 12, (2011), pp. 2922-2924

[9] S. J. Jin and X. J. Tang, "Detection and location of leakage in crude petroleum pipeline", Chinese Journal of Scientific Instrument, vol. 18, no. 4, (1997), pp. 343-348.

[10] S. J. Jin, L. N. Wang and J. Li, "Instantaneous negative pressure wave recognition method in leak detection of crude petroleum transported pipeline", Journal of Electronic Measurement and Instrument, vol. 12 , no. $1,(\mathbf{1 9 9 8})$, pp. 59-64.

[11] Y. C. Jiang and H. X. Wu, "Design and implementation of leak detection in oil pipeline using SCADA system", Instrument Technique and Sensor, vol. 2, (2004), pp. 24-25, 42.

[12] J. J. Xu, C. Liu and S. D. Wang, "Research of small pipeline leak detection applied SPRT algorithm", Electric Machines and Control, vol. 13, no. 3, (2009), pp. 448-451, 457.

[13] E. B. Liu, C. J. Li and C. B. Peng, "Leakage detection for oil pipeline based on negative pressure wave theory", Journal of Harbin Institute of Technology, vol. 41, no. 11, (2009), pp. 285-287.

[14] H. Y. Yang, H. Ye and G. Z. Wang, "The study and development of fault detection for dynamic systems based on chaos theory", Automation \& Instrumentation, vol. 1, (2007), pp. 1-4.

[15] J. H. Liu, H. G. Zhang and J. Feng, "On-line leak-detection method for pressure time series of oil pipeline", Journal of Northeastern University (Natural Science), vol. 30, no. 3, (2009), pp. 321-324.

[16] H. Y. Yang, H. Ye and G. Z. Wang, "Correlation dimension analysis in leak diagnosis for oil pipelines", J. Huazhong Univ. of Sci. \& Tech. (Natural Science Edition), vol. 37, no. 4, (2009), pp. 126-130.

[17] Y. F. Yang and M. J. Wu, "Parameters selection for calculating largest lyapunov exponent from small data sets", Journal of Vibration, Measurement \&Diagnosis, vol. 32, no. 3, (2012), pp. 371-374.

[18] X. Z. Wei and Y. M. Ren, "Forecasting and control of water demand based on the improved largest Lyapunov exponent algorithm”, Journal of Harbin Institute of Technology, vol. 40, no. 1, (2008), pp. 25-27.

[19] J. J. Xu, Y. F. Zhang and P. Wang, "A Kind of Turbine Fault Diagnosis based on Fish-swarm Algorithm", Energy Education Science and Technology Part A: Energy Science and Research, vol. 32, no. 2, (2014), pp. 1325-1330.

[20] Z. L. Liu, J. J. Xu and H. Y. Li, "A kind of energy saving controller based on Y/A change-over", Sensors and Transducers, vol. 161, no. 12, (2013), pp. 331-336.

[21] L. M. Yan, Y. B. Xie and J. J. Xu, "Improved Forward and Backward Substitution in Calculation of Power Distribution Network with Distributed Generation", Journal of Xi'an Jiaotong University, vol. 47, no. 6, (2013), pp. 117-123. 